\title{
Clinical Efficacy and Rehabilitation of Microscopic "Over the Top" for Bilateral Decompression in Degenerative Lumbar Stenosis: A Retrospective Study
}

\author{
Bin Lv $\left(\mathbb{D},{ }^{1}\right.$ Sixin Sun, ${ }^{2}$ Haosheng Wang, ${ }^{1,3}$ Li Xiao, ${ }^{1}$ Tao Xu, ${ }^{4}$ Peng Ji, ${ }^{1}$ Jishan Yuan, ${ }^{1}$ \\ Hua Ding, ${ }^{1}$ Jun Xie, ${ }^{1}$ Nan Meng, ${ }^{1}$ Lei Zhang, ${ }^{1}$ Minjie Hu, ${ }^{1}$ Qinyi Jiang ${ }^{\mathbb{D}},{ }^{1}$ Lei Wang $\mathbb{D}^{1}$ \\ and Xiang Yao $\mathbb{D}^{1}$ \\ ${ }^{1}$ Department of Orthopaedics, The Affiliated People's Hospital of Jiangsu University, Zhenjiang, Jiangsu Province 212002, China \\ ${ }^{2}$ Department of Orthopaedics, The Affiliated Taixing People's Hospital of Yangzhou University, Taixing, \\ Jiangsu Province 225400, China \\ ${ }^{3}$ Department of Orthopaedics, The Second Hospital of Jilin University, Changchun, Jilin Province 130041, China \\ ${ }^{4}$ Department of Orthopaedics, The First Affiliated Hospital of Nanjing Medical University, Nanjing, Jiangsu Province 210029, China
}

Correspondence should be addressed to Qinyi Jiang; njmujqy@sina.com, Lei Wang; wanglei0505779@126.com, and Xiang Yao; yaoxiang6266@163.com

Received 11 May 2020; Revised 2 September 2020; Accepted 27 October 2020; Published 9 December 2020

Academic Editor: Alberto Grassi

Copyright (C) 2020 Bin Lv et al. This is an open access article distributed under the Creative Commons Attribution License, which permits unrestricted use, distribution, and reproduction in any medium, provided the original work is properly cited.

Background. Recently, "over the top" (also called ULBD; microscopic unilateral laminotomy for bilateral decompression) is a less invasive technique for symptomatic degenerative lumbar spinal stenosis (LSS), and this minimally invasive surgical technique has demonstrated favorable therapeutic outcomes. However, the risk of postoperative complications remains controversial. Objective. This study is aimed at determining the clinical efficacy and complication and rehabilitation of the microscopic "over the top" for degenerative LSS in geriatric patients. Study Design. This was a retrospective study. Setting. All data were obtained from the People's Hospital of a University. Methods. A retrospective analysis of 39 consecutive elderly patients treated for LSS by microscopic "over the top" between January 2016 and January 2018 was performed. A postoperative rehabilitation program for geriatric patients with restricted weight-bearing was instituted after the microscopic "over the top" treatment. Estimated blood loss, duration of operation, length of hospitalization, and total complications were also evaluated. The CT and MRI examinations of the lumbar spine were collected to evaluate the completeness of the nerve decompression. Clinical data were assessed at 6 months and 12 months after operation utilizing the visual analog scale (VAS), Oswestry Disability Index (ODI), and 36-Item Short-Form Health Survey (SF-36). Preoperative comorbidities, complications, and revision surgery were also recorded. Results. We enrolled a total of 39 degenerative LSS patients (27 male and 12 female patients, mean age of $75.8 \pm$ 9.2 years). Twenty patients had one-level of degenerative LSS; thirteen patients had two-level of LSS; six patients had threelevel of LSS. The average follow-up time in our study was $14.6 \pm 7.8$ months (range, 6-24 months). The overall complication rate was $10.2 \%(4 / 39)$, and the reoperation rates at one year were $2.5 \%(1 / 39)$. VAS back and leg pain score at 6 months were decreased to $1.8 \pm 0.7$ and $1.4 \pm 0.6$, respectively, and remained at $1.9 \pm 0.3$ and $1.2 \pm 0.2$ at 12 months, respectively. ODI scores improved significantly from $32.26 \pm 6.82$ to $11.44 \pm 2.50$ at 6 months and $10.56 \pm 2.29$ at 12 months. 36-Item Short-Form Health Survey scores revealed a significant improvement throughout follow-up. Postoperative complications included dural tear $(n=2)$, neurologic deficit $(n=1)$, and reoperation $(n=1)$. No infections or hematomas were reported. Limitation. Multicenter research is recommended to confirm our results and investigate the factors related to clinical and radiographic results. Conclusions. Microscopic "over the top" technique is a safe, effective option in the therapy of degenerative LSS and obtained satisfactory functional outcomes when coupled with aggressive rehabilitation, with a long recurrence-free recovery. 


\section{Introduction}

The prevalence of degenerative LSS is expected to increase in the context of an increasing and aging population. Degenerative LSS is the most condition that causes clinical symptoms, such as lower back pain, leg pain, intermittent claudication, and neurological disorders. Symptomatic LSS can have a serious impact on functionality and quality of life and remains the most common indication for surgical intervention on the spine $[1,2]$. The primary objective of surgical intervention for LSS is decompression of the symptomatic neural elements and to preserve or achieve mechanical stability [3].

Conventional laminectomy is a common surgical technique for providing adequate bony decompression and gets good-to-excellent outcomes in $64 \%$ of patients [4]. However, the destruction of the midline structures and the supra/interspinous ligament caused by this treatment result in postoperative destabilization; it also leads to facet joint injury. Therefore, many geriatric patients are reluctant to undergo conventional laminectomy, based on the understanding that geriatric patients are at high risk for serious postoperative complications and poor outcomes.

Recently, "over the top" (also called ULBD; microscopic unilateral laminotomy for bilateral decompression) was initially described by Young et al. [5]. This surgical technique employs a unilateral exposure and muscle retraction, thus minimizing injury to the paraspinal muscles and the spinous process/interspinous ligament midline tension band structures [6] (Figure 1). This technique markedly reduces softtissue damage and intraoperative blood loss. Patients suffer from decreased postoperative pain and can usually be mobilized and discharged early.

Since geriatric patients are at a high risk for comorbidities, it is important to choose appropriate surgical interventions for this age group. For this reason, an appropriate selection of surgical technique should be used to answer two major problems: (1) Does this surgical technique decrease morbidity and mortality rates? (2) Does this surgical technique decompress the neural elements adequately to relieve symptoms? Therefore, this study is aimed at evaluating complication rates and clinical outcomes after a microscopic "over the top" approach in geriatric patients.

\section{Methods}

The study was approved by the Ethics Committee of the Jiangsu University and was performed in accordance with the Declaration of Helsinki. We retrospectively collected medical records of patients who underwent microscopic "over the top" lumbar decompression surgery between January 2016 and January 2018. All surgeries were performed using the "over the top" technique by one senior chief surgeon. Inclusion criteria included (01) 65 years of age or older; (2) patients with LSS confirmed by concordant imaging. Exclusion criteria were as follows: (1) a previous history of LSS surgery; (2) spinal instability, lumbar spondylolisthesis, and overweight patients with a body mass index (BMI) over 40; (3) psychiatric disorders such as schizophrenia, schizoaf- fective disorder, bipolar disorder and major depressive disorder, and peripheral neurological disease; (4) severe heart, lung, kidney, or liver disease. This study included 39 geriatric patients who received conservative treatment option before operation. Surgery was indicated if the conservative treatment had proved ineffective. The details of this study were explained to the patients, and all patients provided informed consent. Demographic and preoperative data (Table 1), including medical history, BMI, comorbidities, and American Society of Anesthesiologists (ASA) score, were documented. To simplify the presentation of medical history, we classified patients' comorbidities into several categories: cardiac, gastrointestinal, renal, pulmonary, and multiple comorbidity. Surgical data included the number of operated level, procedure time, and bleeding. Clinical outcomes included length of hospitalization, postsurgical complications, and revision surgery rate. The preoperative and postoperative cross-sectional area of the lumbar spinal canal was calculated from computed tomography (CT).

Flexion-extension lumbar radiographs were obtained again at 6 and 12 months postoperatively, and spinal instability was defined as a sagittal plane translation of $\geq 5 \mathrm{~mm}$ on flexion-extension radiographs (Radiological evaluation was judged by two observers). Postoperative follow-up consisted of the visual analog scale (VAS), Oswestry Disability Index (ODI) questionnaire, and 36-Item Short-Form Health Survey (SF-36) at 6 and 12 months. Patient-reported outcomes were collected via face-to-face assessment or telephone interviews.

2.1. "Over the Top" Technique. The microscopic decompression procedure has been previously described [7, 8]. In brief, we made a $3-5 \mathrm{~cm}$ midline incision after fluoroscopic confirmation of the surgical level. After skin incision, the multifidus muscle was dissected unilaterally from the spinous process and lamina using a Cobb elevator and retracted by a Taylor retractor. After detachment of paraspinal muscles, ipsilateral laminotomy was performed using a burr and Kerrison punches, followed by flavectomy using a microscope. To view the contralateral side, the operation table and microscope were tilted approximately 15 to $25^{\circ}$. For decompression, undercutting of the spinous process and contralateral lamina was performed using a burr and Kerrison punches, followed by flavectomy. After contralateral laminotomy and flavectomy, complete neural decompression was confirmed by the restoration of dural pulsation (Figure 2).

2.2. Rehabilitation. To obtain satisfactory lumbar functions, a systematic program of earlier active rehabilitation was carried out. The functional outcome analyses were performed by using a visual analog scale (VAS) for low back and leg pain, Oswestry Disability Index (ODI), and the modified MacNab criteria. Patient-reported outcomes were collected via face-to-face assessment or by using telephone interviews.

2.3. Statistics. All statistical analyses were carried out using SPSS software (Chicago, IL, USA). The Kolmogorov-Smirnov test was used to determine whether the distributions 

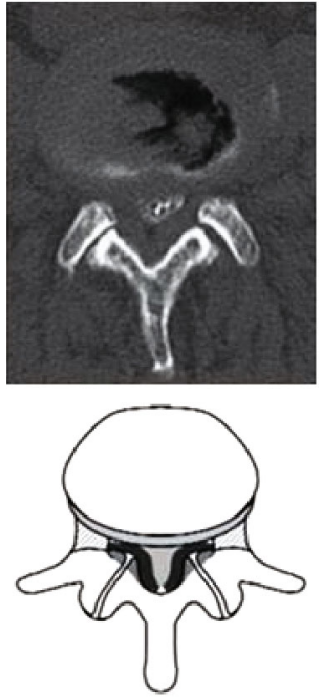

(a)
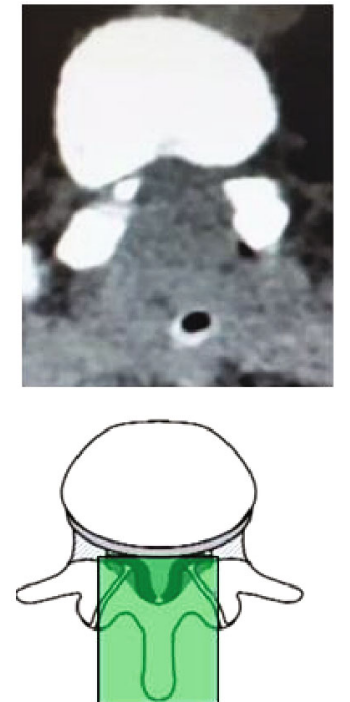

(b)
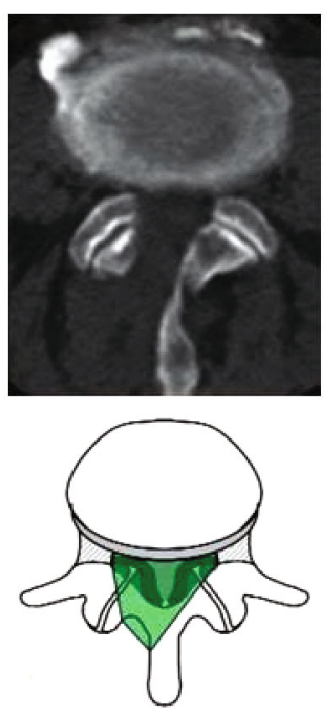

(c)

FIGURE 1: CT scans of traditional decompression and microscopic "over the top" procedures. (a) Spinal lumbar canal stenosis. (b) Traditional decompression approach removed most posterior elements and resected a large portion of the bilateral facet joints. (c) Microscopic "over the top" indicated that a laminotomy was performed by removing a portion of the superior and inferior laminae at the segment, and a small part of the medial facet. Deep cortical surface of contralateral lamina was undercut and drilling was extended to the contralateral medial facet.

TABLE 1: Baseline characteristics of the included patients.

\begin{tabular}{lc}
\hline Characteristics & $(n, \%$, mean \pm SD) \\
\hline Age & $75.8 \pm 9.2$ \\
Gender $($ female/male) & $12 / 27$ \\
BMI $\left(\mathrm{kg} / \mathrm{m}^{2}\right)$ & $23.8 \pm 2.3$ \\
Number of stenotic levels & \\
One level & $20(51.3 \%)$ \\
Two levels & $13(33.3 \%)$ \\
Three levels & $6(15.4 \%)$ \\
Laterality & \\
Unilateral & $17(43.6 \%)$ \\
Bilateral & $22(56.4 \%)$ \\
Level of stenosis & \\
L2 L3 & $4(10.3 \%)$ \\
L3 L4 & $9(23.1 \%)$ \\
L4 L5 & $16(41 \%)$ \\
L5 S1 & $10(25.6 \%)$ \\
Comorbidity & \\
Cardiac & $10(25.6 \%)$ \\
Endocrine & $7(17.9 \%)$ \\
Metabolic & $5(12.8 \%)$ \\
Gastrointestinal & $4(10.3 \%)$ \\
Pulmonary & $6(15.4 \%)$ \\
Renal & $2(5.1 \%)$ \\
Multiple comorbidity & $7(17.9 \%)$ \\
FSA score & $2.72 \pm 0.76$ \\
\hline & $14.6 \pm 7.8$ \\
\hline
\end{tabular}

were significantly different. Data are shown as mean \pm SD (standard deviation), the median (maximum-minimum) for ordinal variables, and the frequency with percent for categorical variables. Significant differences between groups were assessed using a one-way analysis of variance. The significant differences between median values were test by the Kruskal-Wallis test. Categorical comparisons were the chi-square test. $P<0.05$ was considered significant.

\section{Results}

There were 27 (69.2\%) men, and 12 (30.7\%) women patients with a mean age of $75.83 \pm 9.16$ (range, 65-87) years were included in the study. Follow-up ranged from a minimum of 6 to 24 months (mean $14.6 \pm 7.8$ months). Twenty patients had one level of spinal stenosis; thirteen patients had two levels of stenosis; six patients had three levels of stenosis. Postoperative complications included dural tear $(n=2)$, neurologic deficit $(n=1)$, and reoperation $(n=1)$. No infections or hematomas were found in our study. Baseline characteristics of patients are in Table 1. Duration of operation, blood loss, length of hospitalization, and total complications were recorded in Table 2 . There were four complications, including two dural tear (repaired primarily), one neurologic deficit (postoperative weakness of great toe dorsiflexion on the contralateral operative side, which was relieved spontaneously), and one symptom was no improvement (reoperation with laminectomy and fusion).

3.1. Radiographic Analysis. CT scans demonstrated that the estimate of the cross-sectional area of the spinal canal was more significantly larger than preoperative data. Mean postoperative lateral recess height was higher than preoperative 


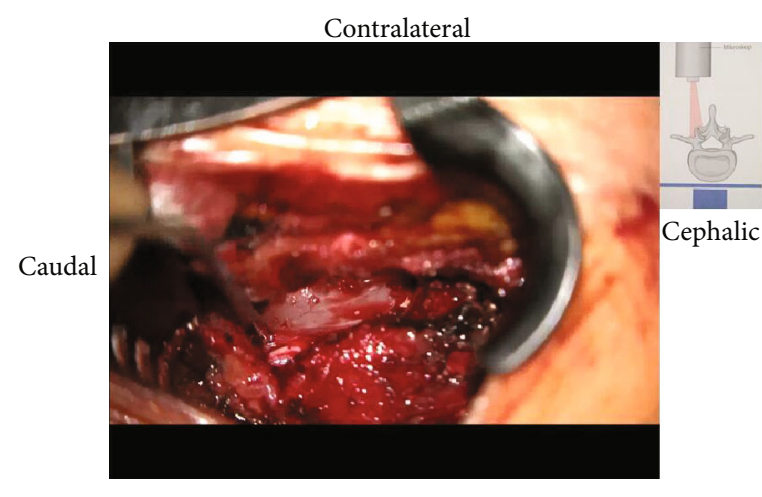

(a)

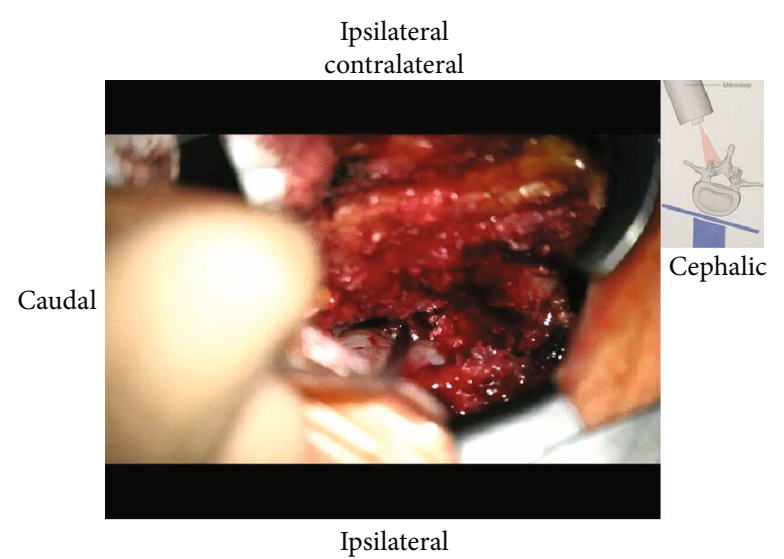

(b)

FIGURE 2: ULBD for spinal lumbar canal stenosis. (a) The inferior half of the L4 lamina has been drilled and the base of the spinous process to expose the ligamentum flavum bilaterally. (b) Decompression of the contralateral side. The tubular retractor is angled beneath the spinous process.

TABLE 2: The perioperative data and complications.

\begin{tabular}{lc}
\hline Parameter & Value $(n, \%$, mean \pm SD) \\
\hline Duration of operation $(\mathrm{min})$ & \\
1-level op & $45.2 \pm 9.4$ \\
2-level op & $88.5 \pm 17.4$ \\
3-level op & $133.6 \pm 37.5$ \\
Estimated blood loss (ml) & \\
1-level op & $80.5 \pm 12.8$ \\
2-level op & $145.7 \pm 55.7$ \\
3-level op & $279.4 \pm 70.3$ \\
Length of hospitalization $(\mathrm{d})$ & $5.4 \pm 1.9$ \\
Total complications $(n /$ rate $)$ & $4(10.3 \%)$ \\
Dural tear & $2(5.1 \%)$ \\
Neurologic deficit & $1(2.6 \%)$ \\
Reoperation & $1(2.6 \%)$ \\
\hline
\end{tabular}

data (Figures 3 and 4); these results were statistically not significant at the follow-up period (Table 3). No abnormal movement in sagittal planes was observed on flexionextension lumbar radiographs at 6 and 12 months (Table 3).

3.2. Clinical Outcomes. Low back and leg pain VAS scores demonstrated significant improvements in outcomes at the mean pre- and postoperative (3, 6, and 12 months) (Table 4). However, these improvements were not significant during the follow-up period. ODI scores decreased significantly from a preoperative score of $31.37 \pm 8.61$ to $12.44 \pm$ 3.50 at 6 months and $12.30 \pm 2.67$ at 12 months, (Table 4 ), and we did not detect the significant differences at 6 months and 12 months.

SF-36 scores demonstrated a significant improvement at 6 months and 12 months after operation except for emotional role. There was no significant difference at both 6 and 12 months (Table 4).

\section{Discussion}

Low back pain (LBP) is the leading health issue of disability globally in the elderly population. Degenerative LSS is one of the most common causes of this condition. Raffo and Lauerman [7] showed that decompression contributed to the greater efficacy than nonsurgical treatments for symptomatic LSS patients in a randomized trial. However, age has been investigated as an independent risk factor for degenerative LSS, which was related to an increased risk of morbidity after open spine surgery [9]. Recent studies have shown that morbidity and complication rates were higher in geriatric patients compared with younger patients after surgery due to a variety of medical comorbidities $[10,11]$. Therefore, it is important to choose appropriate surgical procedure, especially in geriatric patients, for whom the surgical challenges are their psychological and physiological factors. Thus, minimally invasive and efficient surgical intervention was adopted to decrease morbidity and mortality rates.

MIS approaches may produce less muscle-splitting to gain access to the spine and leave the midline structures intact, reducing intraoperative blood loss and relieving postoperative pain [12]. "Over the top" technique used the microendoscopic tubular-retractor system to preserve the facet joints and neural arch of the contralateral side, limiting postoperative destabilization and protecting the neural structures from extensive trauma. Ang et al. [13] retrospectively reviewed the clinical outcomes of 113 patients who underwent "over the top" and found that this technique was associated with reduced blood loss, shorter hospital stay, and lower complication rate. Although studies have confirmed similar results [14], the radiographic outcome and complications for elderly LSS patients have rarely been reported.

In the current study, the average age of patients was 75.83 years, and nearly half of our patients had two levels or multilevel LLS. We found that "over the top" technique was associated with significant improvement in VAS score, ODI score, and SF-36 at the postoperative follow-up period in most patients. Moreover, patients tolerated the surgical 


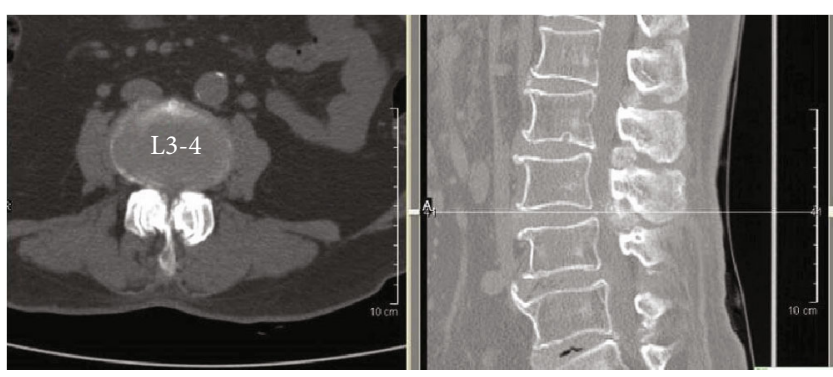

(a)
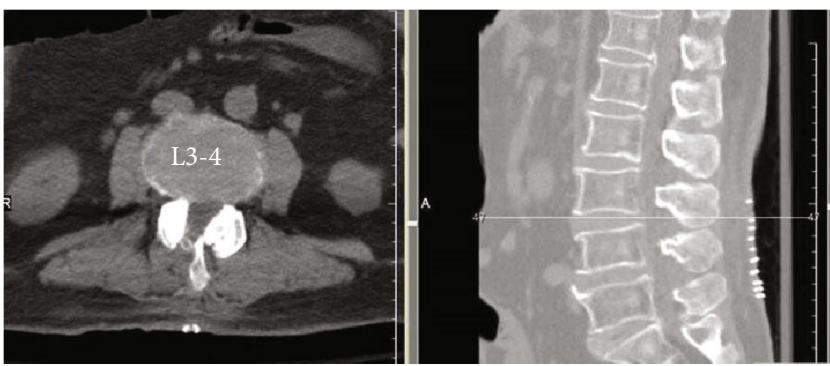

(c)
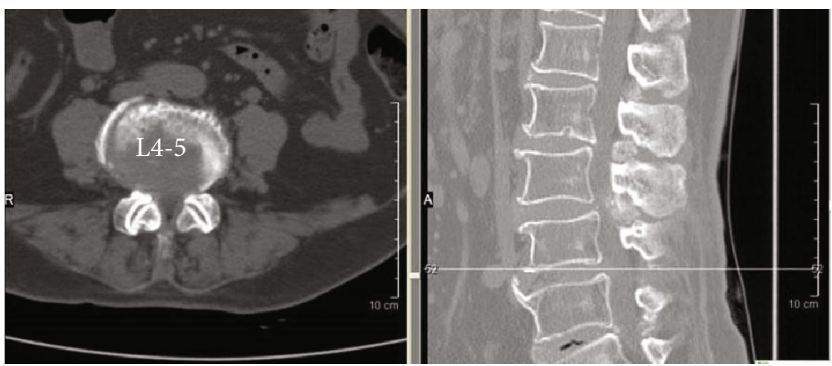

(b)
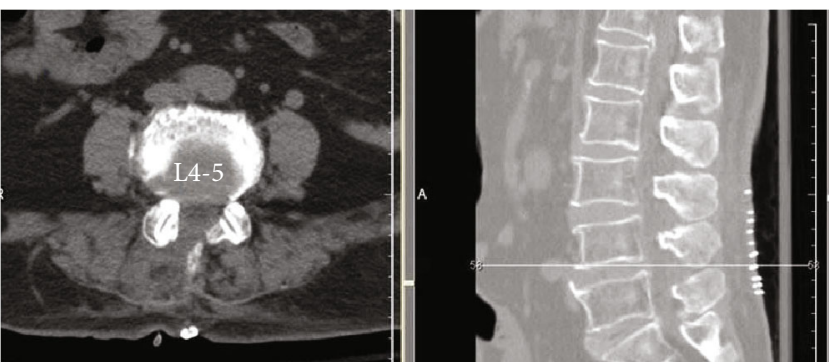

(d)

Figure 3: Preoperative computed tomography of L3-L4, L4-L5 stenosis (a, b), and postoperative computed tomography (c, d) obtained in one patient undergoing ULBD for 2-level stenosis.

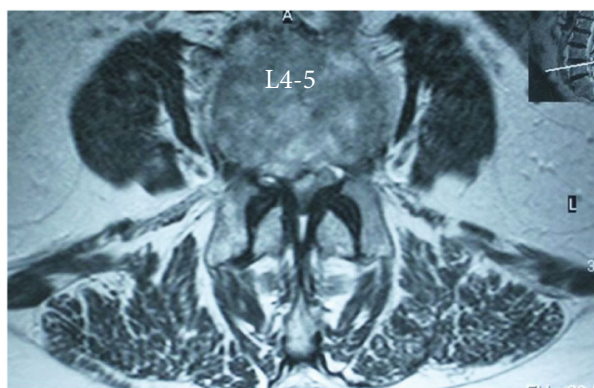

(a)

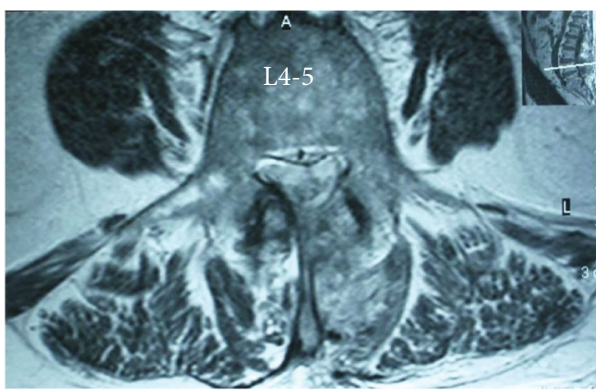

(b)

FIgURE 4: Preoperative (a) and postoperative (b) T2-weighted magnetic resonance imaging of L4-L5 stenosis.

TABle 3: Comparison of radiographic data preoperatively, at 6 months follow-up, and at 12 months follow-up for patients (mean \pm SD).

\begin{tabular}{|c|c|c|c|c|}
\hline & Preoperation & $6 \mathrm{~m}$ & $12 \mathrm{~m}$ & $P$-value \\
\hline Cross-sectional area $\left(\mathrm{mm}^{2}\right)$ & $55.8 \pm 26.6$ & $138.8 \pm 30.5$ & $135.2 \pm 35.7$ & $\begin{array}{c}\mathrm{P} 6 \mathrm{~m}-\mathrm{po}=0.001 \\
\mathrm{P} 6 \mathrm{~m}-12 \mathrm{~m}=0.27\end{array}$ \\
\hline Ipsilateral lateral recess height $(\mathrm{mm})$ & $1.8 \pm 0.6$ & $4.6 \pm 2.2$ & $4.4 \pm 1.6$ & $\begin{array}{c}\text { P6 } \mathrm{m}-\mathrm{po}=0.01 \\
\mathrm{P} 6 \mathrm{~m}-12 \mathrm{~m}=0.53\end{array}$ \\
\hline Contralateral lateral recess height $(\mathrm{mm})$ & $2.5 \pm 0.7$ & $4.1 \pm 1.3$ & $4.0 \pm 0.9$ & $\begin{array}{c}\text { P6 } \mathrm{m}-\mathrm{po}=0.01 \\
\mathrm{P} 6 \mathrm{~m}-12 \mathrm{~m}=0.59\end{array}$ \\
\hline Dynamic motion flexion-extension (mm) & $2.2 \pm 1.5$ & $2.3 \pm 1.7$ & $2.5 \pm 1.4$ & $\begin{array}{c}\mathrm{P} 6 \mathrm{~m}-\mathrm{po}=0.72 \\
\mathrm{P} 6 \mathrm{~m}-12 \mathrm{~m}=0.35\end{array}$ \\
\hline
\end{tabular}

P6 m-po compared preoperation with 6 months follow-up; P6 m-12 m compared 6 months with 12 months follow-up.

procedure well, even though some patients had medical comorbidities. Katz et al. [15] found that elderly patients with medical comorbidity and functional disability might not be very positive about treatment after decompression. Trans- feldt et al. [16] reported that the procedural complication rate of open surgical decompression was $21 \%-40 \%$.

In our study, the overall complication rate was $10.2 \%$, and the results showed satisfactory outcomes. We speculated 
TABle 4: Oswestry Disability Index (ODI), visual analog scale (VAS), and 36-Item Short-Form Health Survey scores preoperatively, at 6 months and 12 months follow-up (mean \pm SD).

\begin{tabular}{|c|c|c|c|c|}
\hline & Preoperation & $6 \mathrm{~m}$ & $12 \mathrm{~m}$ & $P$ value \\
\hline ODI & $31.37 \pm 8.61$ & $12.44 \pm 3.50$ & $12.30 \pm 2.67$ & $\begin{array}{c}\mathrm{P} 6 \mathrm{~m}-\mathrm{po}=0.001 \\
\mathrm{P} 6 \mathrm{~m}-12 \mathrm{~m}=0.38\end{array}$ \\
\hline Back pain VAS & $5.4 \pm 1.6$ & $1.8 \pm 0.7$ & $1.9 \pm 0.3$ & $\begin{array}{c}\mathrm{P} 6 \mathrm{~m}-\mathrm{po}=0.001 \\
\mathrm{P} 6 \mathrm{~m}-12 \mathrm{~m}=0.26\end{array}$ \\
\hline Leg pain VAS & $6.6 \pm 2.8$ & $1.4 \pm 0.6$ & $1.2 \pm 0.2$ & $\begin{array}{c}\mathrm{P} 6 \mathrm{~m}-\mathrm{po}=0.001 \\
\mathrm{P} 6 \mathrm{~m}-12 \mathrm{~m}=0.17\end{array}$ \\
\hline SF-36 & & & & \\
\hline Physical function & $58.67 \pm 5.23$ & $72.45 \pm 5.31$ & $71.86 \pm 4.68$ & $\begin{array}{l}\mathrm{P} 6 \mathrm{~m}-\mathrm{po}=0.001 \\
\mathrm{P} 6 \mathrm{~m}-12 \mathrm{~m}=0.51\end{array}$ \\
\hline Physical role & $29.56 \pm 8.77$ & $49.01 \pm 9.22$ & $49.11 \pm 8.75$ & $\begin{array}{c}\text { P6 } \mathrm{m}-\mathrm{po}=0.001 \\
\mathrm{P} 6 \mathrm{~m}-12 \mathrm{~m}=0.69\end{array}$ \\
\hline Body pain & $40.11 \pm 4.11$ & $68.25 \pm 7.53$ & $68.29 \pm 7.75$ & $\begin{array}{c}\mathrm{P} 6 \mathrm{~m}-\mathrm{po}=0.001 \\
\mathrm{P} 6 \mathrm{~m}-12 \mathrm{~m}=0.44\end{array}$ \\
\hline General health & $52.27 \pm 4.25$ & $58.26 \pm 3.13$ & $57.23 \pm 4.54$ & $\begin{array}{c}\text { P6 } \mathrm{m}-\mathrm{po}=0.01 \\
\text { P6 } \mathrm{m}-12 \mathrm{~m}=0.39\end{array}$ \\
\hline Vitality/energy & $41.73 \pm 3.11$ & $58.97 \pm 5.21$ & $59.12 \pm 5.18$ & $\begin{array}{c}\text { P6 } \mathrm{m}-\mathrm{po}=0.01 \\
\mathrm{P} 6 \mathrm{~m}-12 \mathrm{~m}=0.41\end{array}$ \\
\hline Social function & $40.32 \pm 5.41$ & $51.54 \pm 6.27$ & $52.33 \pm 6.09$ & $\begin{array}{c}\text { P6 } \mathrm{m}-\mathrm{po}=0.01 \\
\mathrm{P} 6 \mathrm{~m}-12 \mathrm{~m}=0.36\end{array}$ \\
\hline Emotional role & $63.62 \pm 5.73$ & $63.72 \pm 5.73$ & $63.69 \pm 5.76$ & $\begin{array}{c}\mathrm{P} 6 \mathrm{~m}-\mathrm{po}=0.31 \\
\mathrm{P} 6 \mathrm{~m}-12 \mathrm{~m}=0.40\end{array}$ \\
\hline Mental health & $43.36 \pm 5.80$ & $63.37 \pm 5.77$ & $63.34 \pm 5.82$ & $\begin{array}{l}\mathrm{P} 6 \mathrm{~m}-\mathrm{po}=0.001 \\
\mathrm{P} 6 \mathrm{~m}-12 \mathrm{~m}=0.22\end{array}$ \\
\hline
\end{tabular}

P6 m-po compared preoperation with 6 months follow-up; P6 m-12 m compared 6 months/with 12 months follow-up.

that the good results and few complications resulted from several inherent advantages of the MIS technique. Postsurgical stress response was found to lead to an imbalance in autonomic, endocrine, and immune systems. MIS technique can decrease inflammation and stress response after surgery. It was found to promote cardiovascular adverse events (hypertension, cardiac dysrhythmias, and myocardial infarctions) in the immediate perioperative period [17]. The frail elderly was suffering from multiple comorbidities, and limited physiologic reserve was vulnerable to stress. Therefore, the frail elderly may benefit from MIS procedures which reduce the risk of surgery.

Aging and medical comorbidities, such as cardiovascular diseases, can result in delayed wound healing that may jeopardize patient outcomes either by direct influence on spinal muscle rehabilitation [18]. "Over the top" results in smaller skin incisions that facilitate wound healing and decrease the risk of wound complications in the aging population. MIS procedure also can reduce blood loss [19]. The cardiovascular and pulmonary compensatory mechanisms are of limited capacity in older patients because age decreases the contractility and increases the stiffness of the left ventricle [20]. These alterations may impair the patient from tolerating large volume shifts, which can lead to life-threatening complications in geriatric patients.

The most common type of complication in "over the top" technique was dural tears. It is generally agreed that the opposite lateral spinal canal may require significant dural sac retraction through the unilateral approach, increasing the risk of dural tear or nerve injury. Here, dural tears occurred in 2 patients $(4.4 \%)$ in our study. We used a 6-0 gauge with a tapered needle to repair dural defects in running locking stitch. The paraspinous muscles and overlying fascia were closed in two layers with nonabsorbable suture used in a watertight fashion. Two patients lied flat for 72 hours after operation and no infections and further headaches. The incidence of dural tears was similar to the previous study. Sidhu et al. [19] showed that the incidence of dural tears was $0 \%$ $18 \%$ for the microsurgical "over the top" technique. This rate was comparable to or lower than the incidence of dural tears reported in most series of decompression surgery for LSS [21]. To our knowledge, we cannot recommend this procedure in cases where severe radiculopathy symptoms are observed. Allowing contralateral microscopic visualization and using angled curets to create more space to perform the decompression surgery could reduce the incidence and 
severity of dural tears. When the dural injury happened, it must be meticulously sutured immediately to avoid CSF leakage.

For "over the top" technique, another main goal of surgery was adequate decompression of the neural elements. We utilized CT to measurements of the cross-sectional area of the spinal canal and lateral recess heights, and postoperative CT demonstrated decompression was enough. In contrast to our results, Thomé et al. [22] found that the ULBD was associated with less sufficient decompression than the bilateral laminotomy, even if the difference was not significant. This finding may suggest that the ULBD approach provides a worse view of the contralateral recess due to the limited exposure via a unilateral approach. However, Moisi et al. [23] concluded in their technical note that the ULBD approach could provide better visualization of the contralateral recess. Our selection of the decompression strategy using the ULBD technique was based on patients' symptoms and severity of LSS. For elderly patients with facet hyperplasia, ipsilateral facetectomy was routinely performed to obtain an adequate decompression for foraminal and lateral recess stenosis, it provided enough space and abduction angle to allow the undermining of the ventral aspect of the spinous process and contralateral lamina, and the posterior midline osteoligamentous structures and contralateral ligamentum flavum could be resected to expose the contralateral side, which allow contralateral facetectomy and provide complete decompression of the dural sac and contralateral nerve root.

During lumbar decompression surgery, the extent of preservation of the bilateral facet joint is an important factor for maintaining spinal stability [24]. Traditional standard decompression involves widely facetectomy and removal of the posterior spinal structures. As a result, the approach can lead to postoperative destabilization, which can lead to the need for spinal fusion and in turn is associated with increased comorbidities in geriatric patient. Mariconda et al. [25] showed that high rates of reoperation in open decompression ranged from $11 \%$ to $30 \%$. In our study, we performed adequate decompression of the spinal stenosis, and no lumbar instability was found in the ULBD procedure. Miyazaki et al. [26].reported that the average percent facet joint preservation was significantly smaller than that in conventional decompression surgery. Further research has to be focused on the evolution of the stability of the treated spinal segment after different types of decompression. Microscopic "over the top" preserved $60 \%-83 \%$ of the facet joint on the approach side and $>90 \%$ of the facet joint on the contralateral side. By contrast, the traditional approach retained $\leq 40 \%$ percent of the facet [27]. Therefore, we thought this MIS method can reduce the risk of postoperative spinal instability at the surgical site due to the satisfactory preservation of the facet joint.

\section{Conclusions}

Our experience supports the use of the ULBD as a less invasive technique for symptomatic LSS to conservative treatment that allows for adequate decompression of neural elements at the affected level. Even after symptom resolution, the general health and mental conditions of patients can also be significantly improved. The results of this study indicate that ULBD surgery is a safe and effective treatment for geriatric patients and does not increase the risk of complications.

5.1. Limitations. Our study has several limitations. First of all, we have our study with a limited number of patients, and the sample size was not sufficient to yield substantial effects. Second limitation, we were not able to compare beneficial results from ULBD in this age group with outcomes from those of an open surgical intervention in a similar age-grouped patient cohort. Finally, this was a retrospective study, and the indications for this surgical procedure were limited in patients with LSS. Additional studies with larger samples and longer follow-up periods should be performed to confirm the present results.

\section{Abbreviations \\ ULBD: Unilateral laminotomy for bilateral decompression \\ LSS: Lumbar spinal stenosis \\ VAS: Visual analog scale \\ ODI: Oswestry Disability Index \\ SF-36: 36-Item Short-Form Health Survey \\ ASA: American Society of Anesthesiologists.}

\section{Data Availability}

Data will not be available because consent and ethical approval was not obtained for sharing. Additionally, all available data has been summarized in the text of the manuscript.

\section{Ethical Approval}

Ethical approval for this study was granted by the Affiliated People's Hospital of Jiangsu University, China.

\section{Consent}

All patients or authorized relatives gave informed consent. We have obtained consent for publication.

\section{Conflicts of Interest}

The authors declare that they have no competing interests.

\section{Authors' Contributions}

Bin Lv, Sixin Sun, Haosheng Wang, and Li Xiao contributed equally to this article.

\section{Acknowledgments}

The authors thank Yan Zhou and Tao Liu for radiology preparation. This study was supported by the Science and Technology Planning Social Development Project of Zhenjiang City, Jiangsu Province (SH2018040 and SH2019085), Clinical Medical Science and Technology Development Foundation of Jiangsu University (Grant No: JLY20180047), Clinical Medical Science and Technology Development 
Foundation of Jiangsu University (Grant No: JLY20180271), and the hospital level project of the Affiliated People's Hospital of Jiangsu University (Grant No. 699012).

\section{References}

[1] S. R. Garfin, H. N. Herkowitz, and S. Mirkovic, "Spinal stenosis," Instructional Course Lectures, vol. 49, pp. 361-374, 2000.

[2] J. N. Katz, G. Stucki, S. J. Lipson, A. H. Fossel, L. J. Grobler, and J. N. Weinstein, "Predictors of surgical outcome in degenerative lumbar spinal stenosis," Spine, vol. 24, no. 21, pp. 22292233, 1999.

[3] J. N. Weinstein, T. D. Tosteson, J. D. Lurie et al., "Surgical versus nonsurgical therapy for lumbar spinal stenosis," The New England Journal of Medicine, vol. 358, no. 8, pp. 794-810, 2008.

[4] J. A. Turner, M. Ersek, L. Herron, and R. Deyo, "Surgery for lumbar spinal stenosis. Attempted meta-analysis of the literature," Spine, vol. 17, no. 1, pp. 1-8, 1992.

[5] S. Young, R. Veerapen, and S. A. O'Laoire, "Relief of lumbar canal stenosis using multilevel subarticular fenestrations as an alternative to wide laminectomy: preliminary report," Neurosurgery, vol. 23, no. 5, pp. 628-633, 1988.

[6] Y. Mikami, M. Nagae, T. Ikeda, H. Tonomura, H. Fujiwara, and T. Kubo, "Tubular surgery with the assistance of endoscopic surgery via midline approach for lumbar spinal canal stenosis: a technical note," European Spine Journal, vol. 22, no. 9, pp. 2105-2112, 2013.

[7] C. S. Raffo and W. C. Lauerman, "Predicting morbidity and mortality of lumbar spine arthrodesis in patients in their ninth decade," Spine, vol. 31, no. 1, pp. 99-103, 2006.

[8] K. Schöller, T. Steingrüber, M. Stein et al., "Microsurgical unilateral laminotomy for decompression of lumbar spinal stenosis: long-term results and predictive factors," Acta Neurochirurgica, vol. 158, no. 6, pp. 1103-1113, 2016.

[9] Z. H. Arinzon, B. Fredman, E. Zohar et al., "Surgical management of spinal stenosis: a comparison of immediate and long term outcome in two geriatric patient populations," Archives of Gerontology and Geriatrics, vol. 36, no. 3, pp. 273-279, 2003.

[10] D. Guha, R. F. Heary, and M. F. Shamji, "Iatrogenic spondylolisthesis following laminectomy for degenerative lumbar stenosis: systematic review and current concepts," Neurosurgical Focus, vol. 39, no. 4, article E9, 2015.

[11] H. S. Kim, B. Paudel, J. S. Jang et al., "Percutaneous full endoscopic bilateral lumbar decompression of spinal stenosis through uniportal-contralateral approach: techniques and preliminary results," World Neurosurgery, vol. 103, pp. 201-209, 2017.

[12] U. Spetzger, A. V. Schilling, G. Winkler, J. Wahrburg, and A. König, "The past, present and future of minimally invasive spine surgery: a review and speculative outlook," Minimally Invasive Therapy \& Allied Technologies, vol. 22, no. 4, pp. 227-241, 2013.

[13] C. L. Ang, B. Phak-Boon Tow, S. Fook et al., "Minimally invasive compared with open lumbar laminotomy: no functional benefits at 6 or 24 months after surgery," The Spine Journal, vol. 15, no. 8, pp. 1705-1712, 2015.

[14] B. Storzer and K. J. Schnake, "Microscopic bilateral decompression by unilateral approach in spinal stenosis," European Spine Journal, vol. 25, Suppl 2, pp. 270-271, 2016.
[15] J. N. Katz, S. J. Lipson, G. W. Brick et al., "Clinical correlates of patient satisfaction after laminectomy for degenerative lumbar spinal stenosis," Spine, vol. 20, no. 10, pp. 1155-1159, 1995.

[16] E. E. Transfeldt, R. Topp, A. A. Mehbod, and R. B. Winter, "Surgical outcomes of decompression, decompression with limited fusion, and decompression with full curve fusion for degenerative scoliosis with radiculopathy," Spine, vol. 35, no. 20, pp. 1872-1875, 2010.

[17] C. Bingqian, X. Feng, S. Xiaowen et al., "Modified posterior lumbar interbody fusion using a single cage with unilateral pedicle screws: a retrospective clinical study," Journal of Orthopaedic Surgery and Research, vol. 10, no. 1, p. 98, 2015.

[18] M. Rahman, L. E. Summers, B. Richter, R. Mimran, and R. Jacob, "Comparison of techniques for decompressive lumbar laminectomy: the minimally invasive versus the "classic" open approach," Minimally Invasive Neurosurgery, vol. 51, no. 2, pp. 100-105, 2008.

[19] G. S. Sidhu, E. Henkelman, A. R. Vaccaro et al., "Minimally invasive versus open posterior lumbar interbody fusion: a systematic review," Clinical Orthopaedics and Related Research, vol. 472, no. 6, pp. 1792-1799, 2014.

[20] A. Severn, "Anaesthesia and the preparation and management of elderly patients undergoing surgery," European Journal of Cancer, vol. 43, no. 15, pp. 2231-2234, 2007.

[21] H. M. Mayer, J. List, A. Korge, and K. Wiechert, "Microsurgery of acquired degenerative lumbar spinal stenosis. Bilateral overthe-top decompression through unilateral approach," Orthopade, vol. 32, no. 10, pp. 889-895, 2003.

[22] C. Thomé, D. Zevgaridis, O. Leheta et al., "Outcome after lessinvasive decompression of lumbar spinal stenosis: a randomized comparison of unilateral laminotomy, bilateral laminotomy, and laminectomy," Journal of Neurosurgery. Spine, vol. 3, no. 2, pp. 129-141, 2005.

[23] M. Moisi, C. Fisahn, L. Tkachenko et al., "Unilateral laminotomy with bilateral spinal canal decompression for lumbar stenosis: a technical note," Cureus, vol. 8, article e623, 2016.

[24] R. A. Deyo, B. I. Martin, W. Kreuter, J. G. Jarvik, H. Angier, and S. K. Mirza, "Revision surgery following operations for lumbar stenosis," The Journal of Bone and Joint SurgeryAmerican Volume, vol. 93, no. 21, pp. 1979-1986, 2011.

[25] M. Mariconda, R. Fava, A. Gatto, C. Longo, and C. Milano, "Unilateral laminectomy for bilateral decompression of lumbar spinal stenosis: a prospective comparative study with conservatively treated patients," Journal of Spinal Disorders \& Techniques, vol. 15, no. 1, pp. 39-46, 2002.

[26] M. Miyazaki, Y. Morishita, C. Takita, T. Yoshiiwa, J. C. Wang, and H. Tsumura, "Analysis of the relationship between facet joint angle orientation and lumbar spine canal diameter with respect to the kinematics of the lumbar spinal unit," Journal of Spinal Disorders \& Techniques, vol. 23, no. 4, pp. 242-248, 2010.

[27] K. Sasai, M. Umeda, T. Maruyama, E. Wakabayashi, and H. Iida, "Microsurgical bilateral decompression via a unilateral approach for lumbar spinal canal stenosis including degenerative spondylolisthesis," Journal of Neurosurgery: Spine, vol. 9, no. 6, pp. 554-559, 2008. 